\title{
Decoding Fast-Paced Error-Related Potentials in Monitoring Protocols
}

\author{
Ricardo Chavarriaga, Iñaki Iturrate, Quentin Wannebroucq and José del R. Millán
}

\begin{abstract}
Error-related EEG potentials (ErrP) can be used for brain-machine interfacing (BMI). Decoding of these signals, indicating subject's perception of erroneous system decisions or actions can be used to correct these actions or to improve the overall interfacing system. Multiple studies have shown the feasibility of decoding these potentials in single-trial using different types of experimental protocols and feedback modalities. However, previously reported approaches are limited by the use of long inter-stimulus intervals (ISI $>2 \mathrm{~s}$ ). In this work we assess if it is possible to overcome this limitation. Our results show that it is possible to decode error-related potentials elicited by stimuli presented with ISIs lower than $1 \mathrm{~s}$ without decrease in performance. Furthermore, the increase in the presentation rate did not increase the subject workload. This suggests that the presentation rate for ErrP-based BMI protocols using serial monitoring paradigms can be substantially increased with respect to previous works.
\end{abstract}

\section{INTRODUCTION}

Real time decoding of error-related brain activity has been proposed as a means to improve brain-machine interfaces (BMI) [1]. In particular EEG modulations elicited after erroneous subject actions or feedback have been consistently reported in multiple studies. Furthermore, these modulations, termed error-related potentials, ErrP, can be reliably decoded in single-trial. This provides information about user perception of errors during brain-machine interaction bringing the possibility of correcting such errors [2], [3] or adapting the system to prevent these errors to reocurr [4], [5], [6], [7].

These approaches exploit ErrPs elicited while the subject monitors the behaviour of an external agent upon which he has no control whatsoever [4], [7]. Notwithstanding the encouraging results of these studies, they have consistently used protocols in which the inter-stimulus interval (ISI) is larger than $2 \mathrm{~s}$. In contrast, other BMI approaches, also based on the decoding of stimulus evoked activity, have used much higher presentation rates. This is the case of P300based interfaces [8], [9], or the decoding of visual evoked activity under the rapid-serial visual processing paradigm (RSVP) [10], [11].

The use of low presentation rates poses a clear limitation on the throughput that can be attained using ErrP-based

*This work was supported by the Swiss National Science Foundation NCCR Robotics (http://www.nccr-robotics.ch). I.I. acknowledges support from the "EPFL Fellows" fellowship programme co-funded by Marie Curie, FP7 Grant agreement no. 291771. This paper only reflects the authors' views and funding agencies are not liable for any use that may be made of the information contained herein.

Authors are with the Defitech Chair on Brain-Machine Interface (CNBI), Center for Neuroprosthetics, School of Engineering, Ecole Polytechnique Fédérale de Lausanne, Station 11, CH-1015, Switzerland

\{ricardo.chavarriaga, inaki.iturrate\}depfl.ch \{quentin.wannebroucq, jose.millan\}@epfl.ch

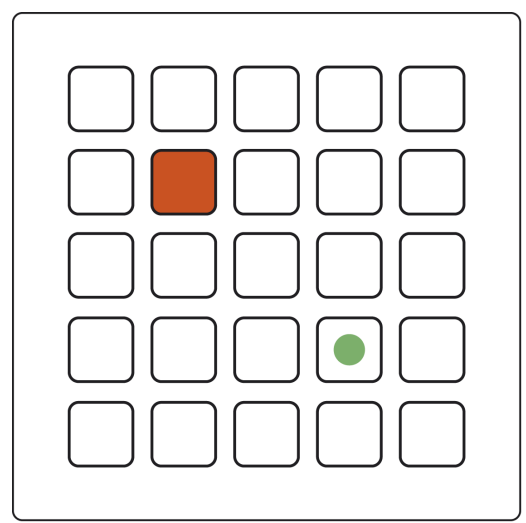

(a)

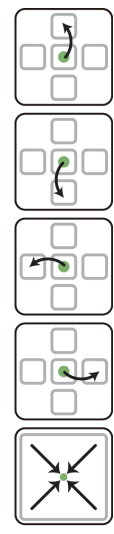

(b)
Fig. 1. Experimental protocol. (a) The subject is presented with a grid of square position, one of which correspond to the target position (red square). A cursor (green circle) can move around these positions or mark one of them as the inferred target location. The subject is asked to monitor whether the cursor's actions are correct or erroneous for reaching the target. (b) Schematic representation of the 5 possible actions corresponding to four directions of movement and a goal-reaching action. Modified from [7].

interaction paradigms. In addition, subjects often report that these protocols are not sufficiently engaging and may even be boring. The rationale of using such long ISIs in ErrPbased protocols is to allow the subject to efficiently assess the agent's behaviour and avoid overlapping EEG activity to hinder single-trial decoding. However, multiple studies have consistently shown that the discriminant activity in these protocols typically appears before $800 \mathrm{~ms}$ after stimulus. This work evaluates ErrP-based paradigms that use shorter ISIs, assessing both the decoding performance and the user appraisal of different presentation rates. Obtained results show that shorter ISIs can be used without diminishing the decoding performance.

\section{METHODS}

\section{A. Experimental protocol}

Six male subjects (mean age $26.3 \pm 3.9$ ) took part in the experiment. All subjects had normal or corrected-tonormal vision and provided informed consent according to the Declaration of Helsinki. The subjects sat comfortably at about $70 \mathrm{~cm}$ from a computer screen that showed the visual stimuli of the protocol. This stimuli consisted of a $5 \times 5$ grid of squares (c.f., Fig. 1). One of them contains a green circle corresponding to a moving cursor and another square is coloured in red indicating that it is the target position. At every step, the cursor can move one position to a neighbouring square (up, down, left or right of its current position). Alternatively, it can perform an additional 
TABLE I

EXPERIMENTAL CONDITIONS. INTER-STIMULUS-INTERVALS USED IN THIS AND PREVIOUS STUDIES.

\begin{tabular}{c|c|c} 
Condition & ISI $[\mathbf{m s}]$ & Length $\mathbf{( 4 0 0}$ trials) \\
\hline Baseline & 1500 & $10^{\prime}$ \\
Fast & 800 & $5^{\prime} 20^{\prime \prime}$ \\
Overlapping & 600 & $4^{\prime}$ \\
\hline
\end{tabular}

\begin{tabular}{c|c|c}
\multicolumn{3}{c}{ Previous studies } \\
\hline Chavarriaga et al., 2010 [4] & 2000 & $13^{\prime} 20^{\prime \prime}$ \\
Chavarriaga et al., 2012 [13] & 3000 & $20^{\prime}$ \\
Iturrate et al., 2013 [14] & {$[3000-3500]$} & $\approx 21^{\prime} 40 "$
\end{tabular}

goal-reaching action indicating target selection. This action was represented by shrinking the ball without changing its position by a factor of $40 \%$ during $400 \mathrm{~ms}$. Subjects were asked to monitor whether the cursor moved towards the target location. It should be noticed that at some locations, several actions would take the cursor closer to the target (e.g., moving up or left in the situation shown in Fig. 1). In these cases, subjects were instructed to consider all these actions as correct. Accordingly, the goal-reaching action was considered correct only if performed at the target location. During the experiment, the cursor moved automatically. At any trial there was a probability of 0.3 for the cursor to perform an erroneous action. To minimise the effect of ocular and muscular artifacts, subjects were instructed to refrain from these movements. Periodical pause periods of $5 \mathrm{~s}$ were included within each run to allow subjects to rest and blink.

Previous studies with this protocol have shown that ErrPs can be reliably decoded with inter-stimulus intervals (ISI) drawn from an uniform distribution in the range [3.0 3.5] s [7]. In order to evaluate if the same results could be obtained with shorter ISIs we tested three conditions summarised in Table I. For sake of comparison, the table also shows the ISIs used in previous studies and the time required in each of them to complete a session of 400 trials. Each subject performed four runs per condition, where each run consisted of 100 trials (i.e. cursor actions). The order of the conditions was chosen pseudorandomly, avoiding testing the same condition consecutively to reduce the effect of fatigue or habituation confounds in the results. After each condition, we applied the NASA-TLX questionnaire to evaluate the subject appraisal of workload for each condition [12]. All recordings were performed in the same session lasting approximately $90 \mathrm{~min}$, including setup.

\section{B. EEG processing and classification}

Neurophysiological signals were recorded using a Biosemi Active Two active acquisition system at a sampling frequency of $2048 \mathrm{~Hz}$, then downsampled to $256 \mathrm{~Hz}$. EEG was acquired with 64 channels following the extended 10/20 montage. To reduce artifact contamination the most peripheral electrodes were excluded from the analysis ${ }^{1}$. Three additional electrodes

${ }^{1}$ The electrodes kept for the analysis were: AF3, AFz, AF4, F5, F3, F1, Fz, F2, F4, F6, FC5, FC3, FC1, FCz, FC2, FC4, FC6, C5, C3, C1, $\mathrm{Cz}, \mathrm{C} 2, \mathrm{C} 4, \mathrm{C} 6, \mathrm{CP} 5, \mathrm{CP} 3, \mathrm{CP} 1, \mathrm{CPz}, \mathrm{CP} 2, \mathrm{CP} 4, \mathrm{CP} 6, \mathrm{P} 5, \mathrm{P} 3, \mathrm{P} 1, \mathrm{Pz}, \mathrm{P} 2$, $\mathrm{P} 4, \mathrm{P} 6, \mathrm{PO} 3, \mathrm{POz}$, and $\mathrm{PO} 4$.
Baseline

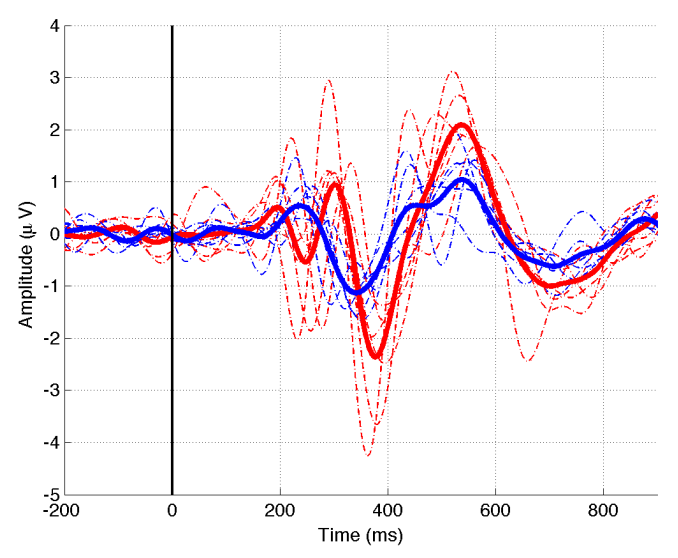

Fast

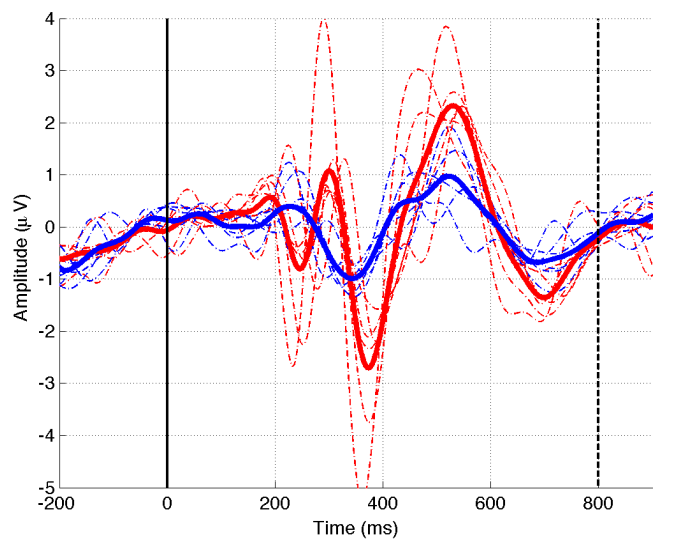

Overlapping

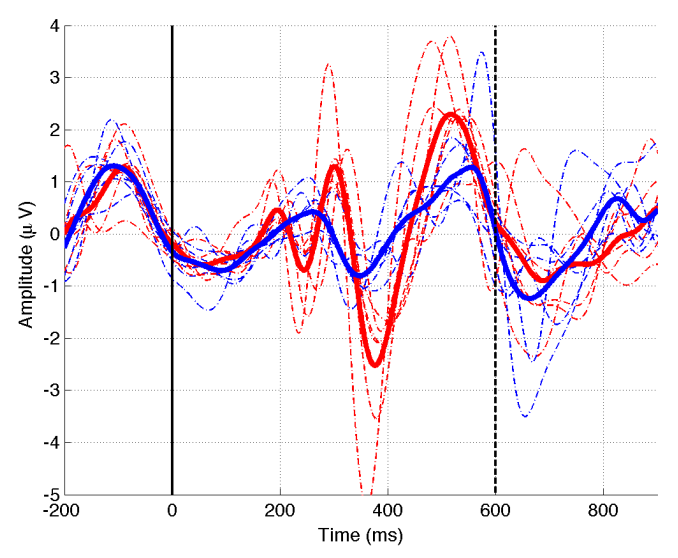

Fig. 2. Grand-average event-related potentials (FCz electrode). Each dashed trace corresponds to the subject grand average ERP for both types of trials (Red: Error; Blue: Correct). Solid lines show the average across subjects. $t=0$ corresponds to the cursor movement onset and the vertical dashed line in (b)-(c) shows the time of the next movement onset.

placed at nasion and on the outer canthi of both eyes were used to derive horizontal and vertical electrooculography (EOG) components [15]. Following previous studies we focused on the frequency range $[210] \mathrm{Hz}$ using a causal 4th order Butterworth filter. EEG was spatially filtered using 


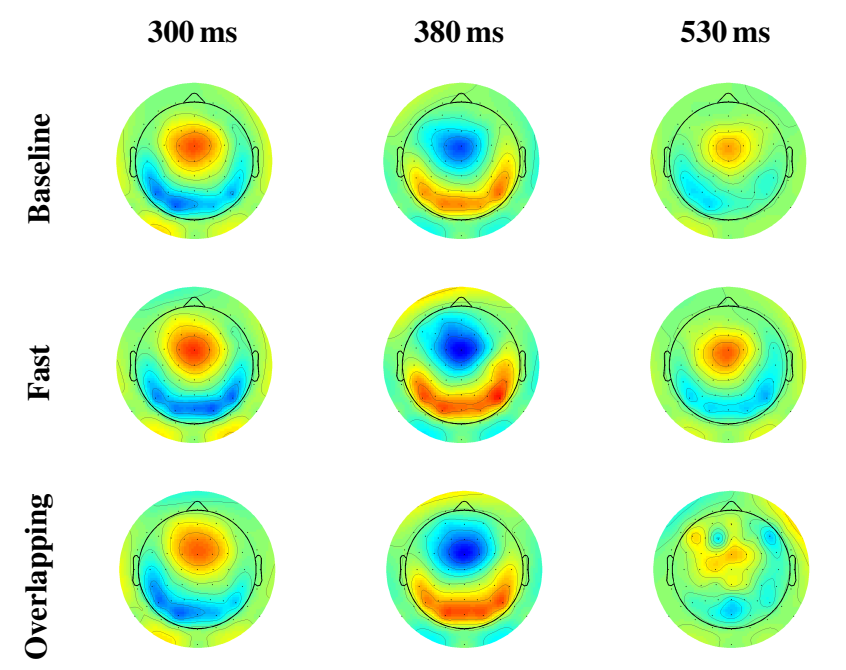

Fig. 3. Topographical representation of the grand average difference ERP (error minus correct conditions) at the times of the two large positive peaks $(t=300 \mathrm{~ms}$ and $t=530 \mathrm{~ms}$ ) and the large negative peak at $t=380 \mathrm{~ms}$. Activity is colour coded from blue to red corresponding to the range $\left[\begin{array}{ll}-2.5 & 2.5\end{array}\right] \mu \mathrm{V}$.

common average reference (CAR). Signals were visually inspected and noisy channels were replaced by linear interpolation of the neighbouring activity.

For the analysis of event-related potentials (ERPs), signals were segmented into epochs corresponding to trials for both error and correct movements. Each epoch comprised the activity from $200 \mathrm{~ms}$ before the cursor movement onset $(t=0)$ until $900 \mathrm{~ms}$ after. For classification purposes, features were extracted from the activity in eight fronto-central electrodes (Fz, $\mathrm{FC} 1, \mathrm{FCz}, \mathrm{FC} 2, \mathrm{C} 1, \mathrm{Cz}, \mathrm{C} 2$, and $\mathrm{CPz}$ ) within the time window [200 600] ms downsampled to $64 \mathrm{~Hz}$. These vectors were then normalised and decorrelated using PCA [14]. To reduce the problem dimensionality we kept the components that explained $95 \%$ of the signal variance in the training set. Trials were classified as erroneous or correct using regularised linear discriminant analysis (LDA) [16]. Performance was assessed using ten-fold cross-validation.

\section{RESULTS}

\section{A. EEG event-related potentials and classification}

EEG activity exhibited the stereotypical modulation in fronto-central areas, as illustrated by the grand average ERPs in Figs. 2 and 3. Activity in the FCz electrode is characterised by a tri-phasic waveform with a first positive ERP component at about $300 \mathrm{~ms}$, followed by a large negativity peaking before $400 \mathrm{~ms}$ and a positive peak between $500 \mathrm{~ms}$ and $600 \mathrm{~ms}$ (c.f., Fig. 2). This pattern has been proposed as a neural correlate of performance monitoring that also comprises the N2-P3 complex as well as the event- and feedback-related negativities (ERN and FRN, respectively) [17].

ERP waveforms for the three conditions are remarkably similar. Notably, clear differences can be observed between the signals elicited by erroneous and correct movements. These results support the hypothesis that the error-related
TABLE II

Classification accuracy. Average across subjects ( \pm SD).

\begin{tabular}{c|c|c|c} 
Condition & Correct & Error & Overall \\
\hline Baseline & $72.0 \pm 6.5$ & $59.9 \pm 6.0$ & $68.4 \pm 6.4$ \\
Fast & $74.8 \pm 5.8$ & $62.1 \pm 6.7$ & $71.1 \pm 5.9$ \\
Overlapping & $76.6 \pm 8.4$ & $60.2 \pm 9.3$ & $70.3 \pm 8.7$
\end{tabular}

modulations of the elicited response are not substantially affected by the presentation rate.

The decoding perfomance is shown in Table II and Fig. 4. We report the overall accuracy, as well as the classification rate for each of the correct and error class separately. As reported in previous studies (c.f., [1]) better decoding performance was obtained for the correct class than for errors. In general, no substantial difference was observed across conditions. It should be noticed that different factors can influence the performance across conditions. On the one hand, it is possible that the Baseline condition is more prone to decreases in the subject's levels of vigilance. On the other hand, shorter ISIs can make the task most difficult to cope.

\section{B. User evaluation}

Subjects provided their appraisal of the experimental protocol by means of the NASA-TLX questionnaire after every run of each condition. The Fast condition had similar workload levels as the Baseline condition despite the fact that stimuli were presented almost twice as fast, see Fig 5 . Initial Fast runs were rated with higher levels at initial runs of the experiment and then decreased. This pattern was even more pronounced in the Overlapping condition, suggesting that subjects were able to adapt to the rapid pace in these conditions.

\section{CONCLUSIONS}

Several BMI protocols are based on the decoding of the EEG activity evoked by the user evaluation of sequentially presented stimuli. This is particularly the case of the P300 and RSVP protocols, and more recently paradigms that exploit ErrPs elicited by action monitoring [1]. However, while P300 and RSVP implementations typically use ISIs ranging from $250 \mathrm{~ms}$ to $100 \mathrm{~ms}$, ErrP-based protocols have mainly used periods of $2 \mathrm{~s}$ or more. Here we show that the signal waveform and decoding performance of ErrPs are not substantially affected by the stimulus presentation rate. This suggests that the ISI in these protocols can be reduced from several seconds to $800 \mathrm{~ms}$ or less. The use of shorter ISI will considerably reduce the calibration time required for these protocols (c.f., Table I), and can potentially result in an increase of the throughput of a BMI system based on this type of signals.

Importantly, user evaluation does not indicate a substantial increase in the workload when shorter ISIs are used, supporting the feasibility of using systems at a faster pace. It is noteworthy that a decrease in the reported workload levels was observed in the Overlapping condition as the experiment went on. This suggests that subjects needed some time to get 
Correct

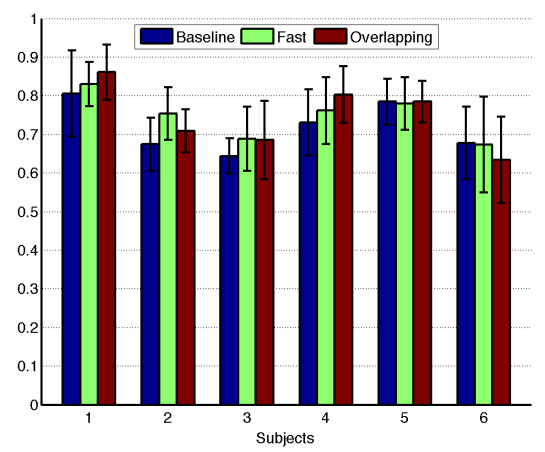

Error

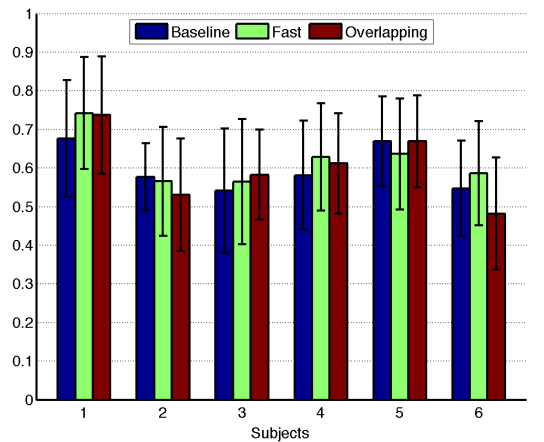

Overall

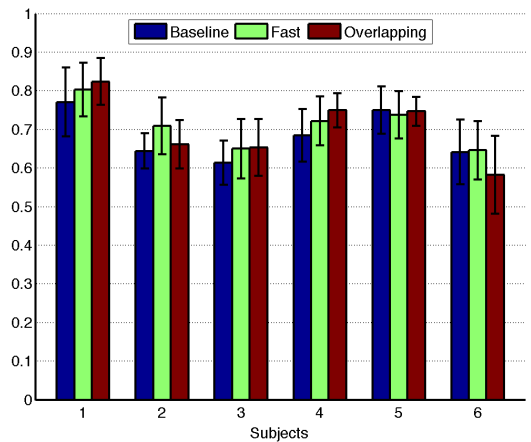

Fig. 4. Classification performance for all subjects and conditions (10-fold cross-validation, mean $\pm \mathrm{SD}$ ).

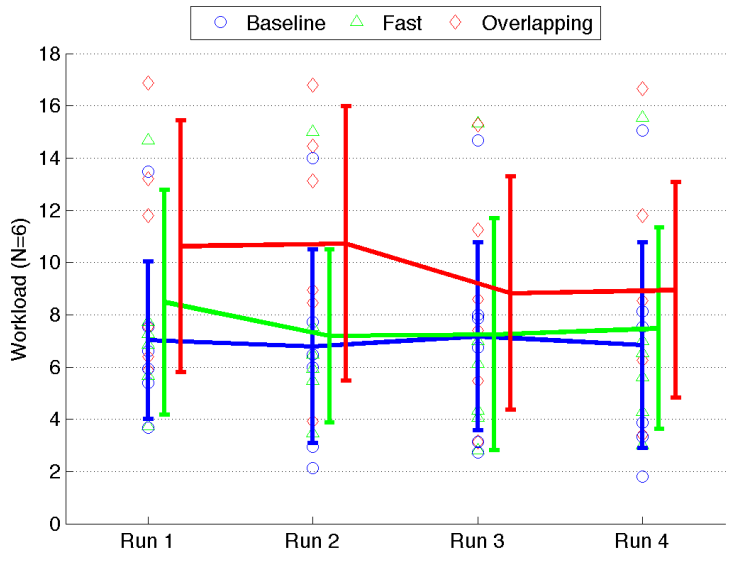

Fig. 5. User workload levels (mean \pm SD across subjects) for each condition and run as indicated by NASA-TLX.

used to the pace in these conditions, before reaching similar workload as in the Baseline condition. Nevertheless, it is yet to be tested whether these conditions will induce higher levels of fatigue in longer experimental sessions.

These results provide further evidence of the robustness of the error-related potentials. They complement studies showing that these signals are stable across time [4], feedback modalities [13] and experimental protocols [14]. Further work is nonetheless required to demonstrate if this robustness actually translates into more reliable BMI applications.

\section{REFERENCES}

[1] R. Chavarriaga, A. Sobolewski, and J. D. R. Millán, "Errare machinale est: the use of error-related potentials in brain-machine interfaces." Front Neurosci, vol. 8, p. 208, 2014. [Online]. Available: http://dx.doi.org/10.3389/fnins.2014.00208

[2] G. Schalk, J. R. Wolpaw, D. J. McFarland, and G. Pfurtscheller, "EEG-based communication: Presence of an error potential." Clin Neurophysiol, vol. 111, no. 12, pp. 2138-2144, Dec 2000.

[3] B. Dal Seno, M. Matteucci, and L. Mainardi, "Online detection of P300 and error potentials in a BCI speller." Comput Intell Neurosci, vol. 2010, p. 307254, 2010. [Online]. Available: http://dx.doi.org/10.1155/2010/307254

[4] R. Chavarriaga and J. Millán, "Learning from EEG error-related potentials in noninvasive brain-computer interfaces." IEEE Trans Neural Syst Rehabil Eng, vol. 18, no. 4, pp. 381-388, Aug 2010. [Online]. Available: http://dx.doi.org/10.1109/TNSRE.2010.2053387
[5] X. Artusi, I. K. Niazi, M.-F. Lucas, and D. Farina, "Performance of a simulated adaptive BCI based on experimental classification of movement-related and error potentials," Emerging and Selected Topics in Circuits and Systems, IEEE Journal on, vol. PP, no. 99, p. 1, 2011.

[6] A. Llera, M. van Gerven, V. Gómez, O. Jensen, and H. Kappen, "On the use of interaction error potentials for adaptive brain computer interfaces," Neural Networks, vol. 24, no. 10 , pp. 1120-1127, Dec 2011. [Online]. Available: http://www.sciencedirect.com/science/article/pii/S0893608011001481

[7] I. Iturrate, L. Montesano, and J. Minguez, "Shared-control braincomputer interface for a two dimensional reaching task using EEG error-related potentials." Conf Proc IEEE Eng Med Biol Soc, vol. 2013, pp. 5258-5262, 2013. [Online]. Available: http://dx.doi.org/10.1109/EMBC.2013.6610735

[8] L. A. Farwell and E. Donchin, "Talking off the top of your head: toward a mental prosthesis utilizing event-related brain potentials," Electroencephalography and Clinical Neurophysiology, vol. 70, pp. 510-523, 1988 .

[9] C. Guger, S. Daban, E. Sellers, C. Holzner, G. Krausz, R. Carabalona, F. Gramatica, and G. Edlinger, "How many people are able to control a P300-based brain-computer interface (BCI)?" Neuroscience Letters, vol. 462, no. 1, pp. 94-98, 2009.

[10] A. Gerson, L. Parra, and P. Sajda, "Cortically-coupled computer vision for rapid image search," IEEE Trans Neural Syst Rehabil Eng, vol. 14, no. 2, pp. 174-179, Jun 2006.

[11] N. Bigdely-Shamlo, A. Vankov, R. R. Ramirez, and S. Makeig, "Brain activity-based image classification from rapid serial visual presentation," IEEE Trans Neural Syst Rehab Eng, vol. 16, no. 5, pp. 432-441, Oct. 2008.

[12] S. Hart and L. Staveland, "Development of NASA-TLX (task load index): Results of empirical and theoretical research," in Human Mental Workload, P. Hancock and N. Meshkati, Eds. North-Holland, 1988, pp. 139-83.

[13] R. Chavarriaga, X. Perrin, R. Siegwart, and J. d. R. Millán, "Anticipation- and error-related EEG signals during realistic humanmachine interaction: A study on visual and tactile feedback," in 34th International Conference of the IEEE Engineering in Medicine and Biology Society (EMBC'12), 2012, pp. 6723-6726.

[14] I. Iturrate, R. Chavarriaga, L. Montesano, J. Minguez, and J. d. R. Millán, "Latency correction of error potentials between different experiments reduces calibration time for single-trial classification," J Neural Engineering, vol. 11, p. 036005, 2014.

[15] R. J. Croft and R. J. Barry, "Removal of ocular artifact from the EEG: A review," Neurophysiologie Clinique, vol. 30, pp. 5-19, 2000.

[16] B. Blankertz, S. Lemm, M. Treder, S. Haufe, and K.R.Müller, "Single-trial analysis and classification of ERP components - a tutorial." Neuroimage, vol. 56, no. 2, pp. 814?-825, 2011. [Online]. Available: http://dx.doi.org/10.1016/j.neuroimage.2010.06.048

[17] M. Ullsperger, A. G. Fischer, R. Nigbur, and T. Endrass, "Neural mechanisms and temporal dynamics of performance monitoring." Trends Cogn Sci, vol. 18, no. 5, pp. 259-267, May 2014. [Online]. Available: http://dx.doi.org/10.1016/j.tics.2014.02.009 